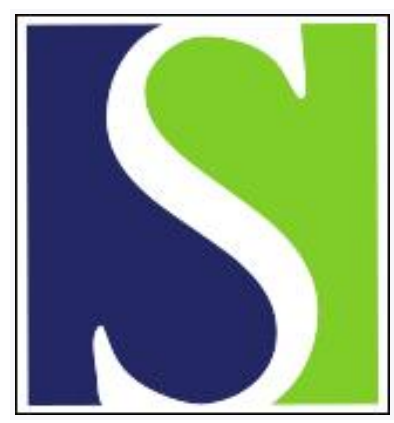

Scand J Work Environ Health 2017;43(2):117-126

https://doi.org/10.5271/sjweh.3612

Published online: 12 Dec 2016, Issue date: 01 Mar 2017

Shift work and overall and cause-specific mortality in the Danish nurse cohort

by Jørgensen JT, Karlsen S, Stayner L, Hansen J, Andersen Z]

Evidence on the association between shift work and mortality is limited. We found significantly enhanced all-cause, cardiovascular and diabetes mortality among nurses with night and evening shifts. We present novel finding of an increase in mortality from Alzheimer's disease and dementia, and potentially psychological disorders among night, evening, and rotating shift workers.

Affiliation: Center for Epidemiology and Screening, Department of Public Health, University of Copenhagen, Copenhagen. Denmark. zorana.andersen@sund.ku.dk

Refers to the following texts of the Journal: 1999;25(2):85-99 2005;31(1):30-35 2009;35(3):163-179 2010;36(2):96-108

The following articles refer to this text: 2017;43(2):97-98; 2017;43(6):569-577; 2020;46(5):508-515; 2022;48(1):31-40

Key terms: all-cause mortality; Alzheimer's disease; cancer; cardiovascular disease; cause-specific mortality; cohort study; Danish nurse cohort; dementia; diabetes; night shift work; nurse; nursing; psychiatric disease; shift work; shift worker

This article in PubMed: www.ncbi.nlm.nih.gov/pubmed/28245504

\title{
Additional material
}

Please note that there is additional material available belonging to this article on the Scandinavian Journal of Work, Environment \& Health -website.

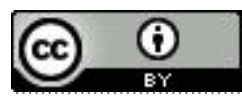




\title{
Shift work and overall and cause-specific mortality in the Danish nurse cohort
}

\author{
by Jeanette Therming Jørgensen, MSc, ${ }^{1}$ Sashia Karlsen, MSc, ${ }^{1}$ Leslie Stayner PhD, ${ }^{2}$ Johnni Hansen, \\ $P h D,{ }^{3}$ Zorana Jovanovic Andersen, $P h D^{1}$
}

\begin{abstract}
Jørgensen JT, Karlsen S, Stayner L, Hansen J, Andersen ZJ. Shift work and overall and cause specific mortality in the Danish nurse cohort. Scand J Work Environ Health. 2017;43(2):117-126. doi:10.5271/sjweh.3612

Objectives Evidence of an effect of shift work on all-cause and cause-specific mortality is inconsistent. This study aimed to examine whether shift work is associated with increased all-cause and cause-specific mortality.

Methods We linked 28731 female nurses (age $\geq 44$ years), recruited in 1993 or 1999 from the Danish nurse cohort where they reported information on shift work (night, evening, rotating, or day), to the Danish Register of Causes of Death to identify deaths up to 2013. We used Cox regression models with age as the underlying scale to examine the associations between night, evening, and rotating shift work (compared to day shift work) and all-cause and cause-specific mortality in models adjusted for potentially confounding variables.

Results Of 18015 nurses included in this study, 1616 died during the study time period from the following causes: cardiovascular disease $(\mathrm{N}=217)$, cancer $(\mathrm{N}=945)$, diabetes $(\mathrm{N}=20)$, Alzheimer's disease or dementia $(\mathrm{N}=33)$, and psychiatric diseases $(\mathrm{N}=67)$. We found that working night [hazard ratio (HR) $1.26,95 \%$ confidence interval $95 \%$ CI) $1.05-1.51$ ] or evening (HR 1.29, 95\% CI 1.11-1.49) shifts was associated with a significant increase in all-cause mortality when compared to working day shift. We found a significant association of night shift work with cardiovascular disease (HR 1.71, 95\% CI 1.09-2.69) and diabetes (HR 12.0, 95\% CI 3.17-45.2, based on 8 cases) and none with overall cancer mortality (HR 1.05, 95\% CI 0.81-1.35) or mortality from psychiatric diseases (HR 1.17, 95\% CI 0.47-2.92). Finally, we found strong association between evening (HR 4.28, 95\% CI 1.62-11.3) and rotating (HR 5.39, 95\% CI 2.35-12.3) shift work and mortality from Alzheimer's disease and dementia (based on 8 and 14 deaths among evening and rotating shift workers, respectively).
\end{abstract}

Conclusions Women working night and evening shifts have increased all-cause, cardiovascular, diabetes, and Alzheimer's and dementia mortality.

Key terms all-cause mortality; Alzheimer's disease; cancer; cardiovascular disease; cohort study; dementia; diabetes; night shift work; nursing; psychiatric disease; shift worker.

It has been suggested that working outside normal work hours, especially at night, has negative health effects $(1,2)$, but evidence of an effect of shift work on allcause mortality is inconsistent (3-12). Several studies $(4-6,10)$, including a recent meta-analysis (13), have reported an increase in all-cause mortality among shift workers, but only a few have detected a statistically significant association $(4,6,13)$ while others have found none $(3,9,12,14)$ or inverse an association (7).

In 2007, an International Agency for Research on Cancer (IARC) working group classified shift work involving circadian disruption as "probably carcino- genic" to humans (group 2A) (15). The majority of recent studies on the health effects of night shift work have focused on female breast cancer incidence (16), and only a few have examined cancer mortality (5-7). One study found significantly increased mortality from any cancer among female but not among male shift workers (5). Few studies have examined shift work and cancer-specific mortality $(6,7,17-19)$. Gu et al (6) found an association with lung cancer mortality and working shifts $\geq 15$ years among American female nurses after adjustment for potential confounders including tobacco smoking. Yong et al (7), found no

1 Center for Epidemiology and Screening, Department of Public Health, University of Copenhagen, Copenhagen, Denmark.

2 Division of Epidemiology \& Biostatistics, University of Illinois at Chicago School of Public Health, Chicago, IL, USA.

3 Danish Cancer Research Centre, Danish Cancer Society, Copenhagen, Denmark.

Correspondence to: Zorana Jovanovic Andersen, Center for Epidemiology and Screening, Department of Public Health, University of Copenhagen, Copenhagen. Denmark. [E-mail: zorana.andersen@sund.ku.dk] 
increase in lung cancer mortality among male chemical shift workers. Carter et al (19) detected an association between rotating shift work and ovarian cancer mortality, whereas Lin et al $(17,18)$ found no association between shift work and either pancreatic or biliary tract cancer mortality.

Shift work has been linked to an increased incidence of coronary or ischemic heart disease (IHD) $(20,21)$ and diabetes $(2,22)$, but there is limited evidence on mortality due to cardiovascular disease (CVD) (23) and diabetes (12). To date, no studies have examined the effect of shift work on Alzheimer's disease, dementia or psychiatric diseases, despite the fact that poor sleep and sleep deprivation have been linked to increased risk of cognitive and neurodegenerative outcomes (24-26). In this study, we examine the association between shift work and all-cause mortality and mortality due to CVD, cancer, diabetes, neurodegenerative and psychiatric diseases in the Danish nurse cohort (DNC).

\section{Methods}

\section{Study population}

The DNC study (27) was initiated in 1993, when 23 170 female members of the Danish Nurses Organization aged $>44$ years were invited to participate in this nationwide study inspired by the American Nurses' Health Study. A total of 19898 (86\%) responded positively. In 1999, the cohort was expanded, adding an additional 10534 nurses aged 44 years, 8833 $(84 \%)$ of whom agreed to participate. Cohort participation involved answering a comprehensive selfadministrated questionnaire on lifestyle, health, use of hormones and occupational characteristics, including working hours and the work environment. Using a unique personal identification number, we linked the DNC to the Danish Civil Registration System (28) in order to obtain information on cohort participants' vital status (death, emigration, disappearance, etc) during follow-up until 2013.

\section{Shift work definition}

Shift work data was self-reported by nurses who were in the workforce at the time of recruitment (excluding those who were retired, on sick leave, or unemployed) and nurses who answered the following question on shift work status: "Do you normally work in: a) day, b) evening, c) night, or d) rotating shifts?". Rotating shifts can be working either day (typically 07:00-15:00 hours) and evening (15:00-23:99 hours) or day, evening and night (23:00-07:00 hours).

\section{Health outcomes}

Information on the deceased cohort participants' causes of death was obtained from the Danish Register of Causes of Death (29), which contains information on all deaths of Danish residents dying in Denmark. Causes of deaths are coded according to the World Health Organization's International Classification of Diseases (ICD) version-10 (after 1994) or ICD-8 (before 1994). All death certificates have underlying cause of death and up to four contributory causes of death, which are not mandatory. We examined all-cause mortality as all deaths occurring during follow-up, including 38 deaths registered in the Civil Registration System with missing cause of death data (no record in the Register of Causes of Deaths). We examined the following cause-specific causes of death using the underlying cause of death: total CVD (ICD-10: I00-99, ICD-8: 4010, 4100, 4129, 4279, 4339, 4369, 4412, 4500), IHD (ICD-10: I20-25, ICD8: 4100, 4129), stroke (ICD-10: I60-69, ICD-8: 4339, 4369), other CVD (ICD-10: I00-09, I26-28, I30-50, I7099, ICD-8: 4279, 4412, 4500), all-cancer (ICD-10: C0097 \& ICD-8: 1578-79, 1538, 1740, 1621, 1830, 2022, 2041), breast cancer (ICD-10: C50, ICD-8: 1740), lung cancer (ICD-10: C33-34, ICD-8: 1621), ovarian cancer ICD-10: C56, C570-574, ICD-8: 1830), pancreatic cancer (ICD-10: C25, ICD-8: 1578-1579), and colorectal cancer (ICD-10: C18-19, C20, C21, ICD-8: 1538). Additionally, we defined the following cause-specific mortality outcomes based on underlying or contributing cause of death: hypertension (ICD-10: I10-15, ICD-8: 4010); diabetes (ICD-10: E10-14), Alzheimer's and dementia, combined (ICD10: F00-01, F03, G30), psychiatric and behavioral diseases, combined (ICD-10: F01, F03-99, ICD-8: 2990, 3032, 3040, 5710, 9779).

\section{Statistical analysis}

Cox proportional hazard regression model with age as the underlying time scale, was used to analyze mortality (all-cause and cause-specific) as a function of shiftwork, in two different models: crude (age-adjusted as age is underlying time scale) and fully adjusted, additionally adjusted for (i) smoking (never/past/current); (ii) packyears [defined as 20 cigarettes/day per year, calculated from smoking intensity (number of cigarettes a day) and smoking duration (years)]; (iii) leisure-time physical activity [categorized in low/medium/high and based on the following question: "Which of the following statements describes you best? (a) Exercise heavily and do competitive sports regularly or several times a week; (b) Do sports/heavy gardening or similar $\geq 4$ hours a week; (c) walk, bike or doing other light exercise $\geq 4$ hours a week; (d) Reading, watching television or other sedentary activities"]; (iv) body mass index (BMI) [cal- 
Table 1. Characteristics of 18015 nurses at baseline (1993 and 1999) by status (active/dead) at end of follow-up (31 December 2012 ).

Total

Person-years

Work type

Day

Evening

Night

Rotating

Body mass index $\left(\mathrm{kg} / \mathrm{m}^{2}\right)$

$<18.5$

$18.5-24.9$

$25-29.9$

$\geq 30$

Smoking status

Never

Past

Current

Number of pack years ${ }^{\mathrm{C}}$

$\leq 10$

$11-20$

$>20$

Never-smokers

Alcohol consumption (drinks/week)

0

$1-14$ (moderate)

$>15$ (heavy)

Physical activity

Low

Medium

High

Diet

Consume vegetables on daily basis

Consume fruit on daily basis

Avoids fatty meat

Self-reported preexisting diseases

Hypertension

Diabetes

Myocardial infarction

Self-reported health

Very good

Good

Moderate

Bad

Very bad

Working status

Working

Homeworker

Retired

Unemployed/rehabilitation

Other

Stressful work environment

Never

Rarely

Occasionally

Often

Almost always

Marital status

Married

Separated

Divorced

Single

Widow

Use of hormone therapy

Never-users

Ever-users

Use of oral contraceptives

Never-users

Ever-users

Births

0

$\geq 1$

All-cause mortality.

b Nurse who were alive and dead at end of follow-up were compared using Pearson's Chi-squared for categorical variables.

${ }^{c}$ One pack year was defined as 20 cigarettes/year in ever-smokers.

16037

\begin{tabular}{|c|c|c|c|c|c|c|}
\hline \multicolumn{2}{|c|}{ Total } & \multicolumn{2}{|c|}{ Alive } & \multicolumn{2}{|c|}{ Dead $^{\text {a }}$} & \multirow[t]{2}{*}{ P-value } \\
\hline $\mathrm{N}$ & $\%$ & $\mathrm{~N}$ & $\%$ & $\mathrm{~N}$ & $\%$ & \\
\hline $\begin{array}{r}18015 \\
316644\end{array}$ & $\begin{array}{l}100 \\
100\end{array}$ & $\begin{array}{r}16399 \\
296468\end{array}$ & $\begin{array}{l}91.0 \\
93.6\end{array}$ & $\begin{array}{r}1616 \\
20176\end{array}$ & $\begin{array}{l}9.0 \\
6.4\end{array}$ & \\
\hline $\begin{array}{r}11272 \\
1805 \\
980 \\
3958\end{array}$ & $\begin{array}{r}62.6 \\
10.0 \\
5.4 \\
22.0\end{array}$ & $\begin{array}{r}10338 \\
1569 \\
829 \\
3663\end{array}$ & $\begin{array}{r}63.0 \\
9.6 \\
5.1 \\
22.3\end{array}$ & $\begin{array}{l}934 \\
236 \\
151 \\
295\end{array}$ & $\begin{array}{r}57.8 \\
14.6 \\
9.3 \\
18.3\end{array}$ & $<0.001$ \\
\hline
\end{tabular}

\begin{tabular}{|c|c|c|c|c|c|c|}
\hline $\begin{array}{r}354 \\
12688 \\
3864 \\
1009\end{array}$ & $\begin{array}{r}2.0 \\
70.4 \\
22.0 \\
5.6\end{array}$ & $\begin{array}{r}291 \\
11593 \\
3613 \\
902\end{array}$ & $\begin{array}{r}1.8 \\
70.7 \\
22.0 \\
5.5\end{array}$ & $\begin{array}{r}63 \\
1095 \\
351 \\
107\end{array}$ & $\begin{array}{r}3.9 \\
67.8 \\
21.7 \\
6.6\end{array}$ & $<0.001$ \\
\hline $\begin{array}{l}6725 \\
5244 \\
6046\end{array}$ & $\begin{array}{l}37.3 \\
29.1 \\
33.6\end{array}$ & $\begin{array}{l}6323 \\
4877 \\
5199\end{array}$ & $\begin{array}{l}38.6 \\
29.7 \\
31.7\end{array}$ & $\begin{array}{l}402 \\
367 \\
847\end{array}$ & $\begin{array}{l}24.9 \\
22.7 \\
52.4\end{array}$ & $<0.001$ \\
\hline $\begin{array}{l}5096 \\
2942 \\
3252 \\
6725\end{array}$ & $\begin{array}{l}28.3 \\
16.3 \\
18.1 \\
37.3\end{array}$ & $\begin{array}{l}4745 \\
2695 \\
2636 \\
6323\end{array}$ & $\begin{array}{l}28.9 \\
16.4 \\
16.1 \\
38.6\end{array}$ & $\begin{array}{l}351 \\
247 \\
616 \\
402\end{array}$ & $\begin{array}{l}21.7 \\
15.3 \\
38.1 \\
24.9\end{array}$ & $<0.001$ \\
\hline $\begin{array}{r}2190 \\
11522 \\
4303\end{array}$ & $\begin{array}{l}12.2 \\
64.0 \\
23.9\end{array}$ & $\begin{array}{r}1929 \\
10607 \\
3863\end{array}$ & $\begin{array}{l}11.8 \\
64.7 \\
23.6\end{array}$ & $\begin{array}{l}261 \\
915 \\
440\end{array}$ & $\begin{array}{l}16.2 \\
56.6 \\
27.2\end{array}$ & $<0.001$ \\
\hline $\begin{array}{r}956 \\
11906 \\
5153\end{array}$ & $\begin{array}{r}5.3 \\
66.1 \\
28.6\end{array}$ & $\begin{array}{r}817 \\
10803 \\
4779\end{array}$ & $\begin{array}{r}5.0 \\
65.9 \\
29.1\end{array}$ & $\begin{array}{r}139 \\
1103 \\
374\end{array}$ & $\begin{array}{r}8.6 \\
68.3 \\
23.1\end{array}$ & $<0.001$ \\
\hline $\begin{array}{l}17787 \\
17387 \\
16470\end{array}$ & $\begin{array}{l}98.7 \\
96.5 \\
91.4\end{array}$ & $\begin{array}{l}16214 \\
15856 \\
15060\end{array}$ & $\begin{array}{r}98.9 \\
96 . \\
91.8\end{array}$ & $\begin{array}{l}1573 \\
1531 \\
1410\end{array}$ & $\begin{array}{l}97.3 \\
94.7 \\
87.3\end{array}$ & $\begin{array}{l}<0.001 \\
<0.001 \\
<0.001\end{array}$ \\
\hline $\begin{array}{r}1890 \\
158 \\
47\end{array}$ & $\begin{array}{r}10.5 \\
0.9 \\
0.3\end{array}$ & $\begin{array}{r}1619 \\
134 \\
32\end{array}$ & $\begin{array}{l}9.9 \\
0.8 \\
0.2\end{array}$ & $\begin{array}{r}271 \\
24 \\
15\end{array}$ & $\begin{array}{r}16.8 \\
1.5 \\
0.9\end{array}$ & $\begin{array}{r}<0.001 \\
0.006 \\
<0.001\end{array}$ \\
\hline $\begin{array}{r}7994 \\
7940 \\
1894 \\
166 \\
21\end{array}$ & $\begin{array}{r}44.4 \\
44.1 \\
10.5 \\
0.9 \\
0.1\end{array}$ & $\begin{array}{r}7463 \\
7170 \\
1624 \\
129 \\
13\end{array}$ & $\begin{array}{r}45.5 \\
43.7 \\
9.9 \\
0.8 \\
0.1\end{array}$ & $\begin{array}{r}531 \\
770 \\
270 \\
37 \\
8\end{array}$ & $\begin{array}{r}32.9 \\
47.6 \\
16.7 \\
2.3 \\
0.5\end{array}$ & $<0.001$ \\
\hline $\begin{array}{r}17924 \\
1 \\
9 \\
4 \\
77\end{array}$ & $\begin{array}{r}99.5 \\
<0.1 \\
<0.1 \\
<0.1 \\
0.4\end{array}$ & $\begin{array}{r}16313 \\
1 \\
8 \\
3 \\
74\end{array}$ & $\begin{array}{r}99.5 \\
<0.1 \\
<0.1 \\
<0.1 \\
0.5\end{array}$ & $\begin{array}{r}1611 \\
0 \\
1 \\
1 \\
3\end{array}$ & $\begin{array}{r}99.7 \\
0.0 \\
0.1 \\
0.1 \\
0.2\end{array}$ & 0.43 \\
\hline $\begin{array}{r}251 \\
2847 \\
7788 \\
5713 \\
1416\end{array}$ & $\begin{array}{r}1.4 \\
15.8 \\
43.2 \\
31.7 \\
7.9\end{array}$ & $\begin{array}{r}205 \\
2579 \\
7072 \\
5245 \\
1298\end{array}$ & $\begin{array}{r}1.3 \\
15.7 \\
43.1 \\
32.0 \\
7.9\end{array}$ & $\begin{array}{r}46 \\
268 \\
716 \\
468 \\
118\end{array}$ & $\begin{array}{r}2.8 \\
16.6 \\
44.3 \\
29.0 \\
7.3\end{array}$ & $<0.001$ \\
\hline $\begin{array}{r}13476 \\
328 \\
2094 \\
1405 \\
712\end{array}$ & $\begin{array}{r}74.8 \\
1.8 \\
11.6 \\
7.8 \\
4.0\end{array}$ & $\begin{array}{r}12404 \\
292 \\
1876 \\
1230 \\
597\end{array}$ & $\begin{array}{r}75.6 \\
1.8 \\
11.4 \\
7.5 \\
3.6\end{array}$ & $\begin{array}{r}1072 \\
36 \\
218 \\
175 \\
115\end{array}$ & $\begin{array}{r}66.3 \\
2.2 \\
13.5 \\
10.8 \\
7.1\end{array}$ & $<0.001$ \\
\hline $\begin{array}{r}13664 \\
4351\end{array}$ & $\begin{array}{l}75.8 \\
24.2\end{array}$ & $\begin{array}{r}12607 \\
3792\end{array}$ & $\begin{array}{l}76.9 \\
23.1\end{array}$ & $\begin{array}{r}1057 \\
559\end{array}$ & $\begin{array}{l}65.4 \\
34.6\end{array}$ & $<0.001$ \\
\hline $\begin{array}{r}5788 \\
12227\end{array}$ & $\begin{array}{l}32.1 \\
67.9\end{array}$ & $\begin{array}{r}5045 \\
11354\end{array}$ & $\begin{array}{l}30.8 \\
69.2\end{array}$ & $\begin{array}{l}743 \\
873\end{array}$ & $\begin{array}{l}46.0 \\
54.0\end{array}$ & $<0.001$ \\
\hline $\begin{array}{r}1978 \\
16037\end{array}$ & $\begin{array}{l}11.0 \\
89.0\end{array}$ & $\begin{array}{r}1720 \\
14679\end{array}$ & $\begin{array}{l}10.5 \\
89,5\end{array}$ & $\begin{array}{r}258 \\
1358\end{array}$ & $\begin{array}{l}16.0 \\
84.0\end{array}$ & $<0.001$ \\
\hline
\end{tabular}


culated from self-reported height and weight $\left.\left(\mathrm{kg} / \mathrm{m}^{2}\right)\right]$; (v) alcohol consumption (number of drinks per week/ none, moderate $1-14$, heavy $\geq 15$ ); (vi) diet ["How often do you eat vegetables and fruits?" (a) Rarely or never, (b) a couple of times a week, (c) daily, or (d) several times a day" and "Do you avoid fatty meat? (yes/no)"]; (vii) pre-existing diseases [based on whether or not participants reported being diagnosed or taking medication to treat the disease hypertension, diabetes or myocardial infarction (MI)]; self-reported health ["How would you evaluate your present state of health? (a) very good, (b) good, (c) moderate, (d) bad, or (e) very bad"]; (viii) work stress ["How often are you so busy that you have difficulties in doing your work tasks? (a) never, (b) rarely, (c) occasionally, (d) often, (e) almost always"]; (ix) marital status (married/separated/divorced/single/ widow); (x) female reproductive factors (a) births ["How many children have you given birth to?", dichotomized into $0 / \geq 1$ ], (b) use of hormone therapy ["Are you or have you previously been on hormone replacement therapy (HRT) with estrogen? (No, I have never been in hormone replacement therapy/I have previously been in hormone replacement therapy/ I am currently in hormone replacement therapy)"], and (c) oral contraceptives ["Have you ever used oral contraceptives? (yes/ no)"]. Analysis of breast and ovarian cancer mortality were additionally adjusted for number of births and age at first birth. Lastly, we examined whether BMI or a stressful work environment acted as a mediating factors in the association between shift work and all-cause mortality, by examining changes in risk estimates with and without adjustment for BMI and work stress in the fully adjusted model.

\section{Results}

Of the 28731 participants in the DNC, 10716 were excluded for the following reasons: (i) emigration prior to cohort baseline ( $\mathrm{N}=4$ ), (ii) retired, unemployed or on sick leave at the time of cohort recruitment $(\mathrm{N}=6721)$, (iii) missing information on shift work schedule ( $\mathrm{N}=669$ ), and (iv) missing information on $\geq 1$ potentially confounding variable(s) $(\mathrm{N}=3322)$. The final analysis comprised 18015 participants. Mean follow-up was 17.6 years, giving a total 316644 person-years, during which 1616 nurses died, including 217 from CVD, 945 from cancer, 20 from diabetes, 33 from Alzheimer's or dementia, and 67 from psychiatric diseases.

A majority of the nurses worked day shifts $(62.6 \%)$, followed by rotating $(22.0 \%)$, evening $(10.0 \%)$ and permanent night $(5.4 \%)$ shifts at the time of recruitment (table 1). Night and evening shift work were more prevalent among nurses who died $(9.3 \%$ and $14.6 \%)$ than among those who were alive $(5.1 \%$ and $9.6 \%)$ at the end of follow-up. Nurses who died were older at the recruitment (mean age 54.2 years) than nurses who were alive at the end of follow-up (mean age 49.9 years). Furthermore, nurses who died smoked more, used HRT more frequently, and less oral contraceptives than nurses who remained alive at the end of follow-up.

Nurses working night shifts were more likely to be current smokers, overweight and obese, and HRT users, but less likely to be married than nurses working other shifts (table 2). Nurses working rotating shifts were more similar to those working day shift than those working night shifts. Mean age [standard deviation (SD)] at baseline was 50.2 (4.7), 51.6 (5.5), 52.9 (5.6) and 49.2 (4.3) years for day, evening, night and rotating shift workers, respectively.

Compared to nurses working day shifts, we found a statistically significant increase in all-cause mortality among nurses working evening [hazard ratio (HR): 1.53, 95\% confidence interval (95\% CI) 1.33-1.77] and night (HR 1.74, 95\% CI 1.48-2.07) shifts, and no increase in those working rotating shifts in the crude model. Estimates were attenuated, but remained statistically significant in the fully adjusted model for evening (HR1.29, 95\% CI 1.11-1.49) and night (HR 1.26, 95\% CI 1.051.51 ) shifts (table 3 ). These estimates were only slightly enhanced when BMI and perceived stress at work were left out of the fully adjusted model (results are available in supplemental material table $\mathrm{C}$, http://www.sjweh.fi/ index.php?page=data-repository). We found a statistically significant increase in CVD mortality among nurses working night shifts (HR 1.71, 95\% CI 1.09-2.69) and a weaker, statistically non-significant increase among nurses working evening (HR 1.47, 95\% CI 0.98-2.18) and rotating (HR 1.24, 95\% CI 0.87-1.77) shifts in the fully adjusted model. We found no associations between cancer mortality and evening (HR 1.15, 0.95-1.40), night (HR 1.05, 95\% CI 0.81-1.35), or rotating shift (HR 0.91, $95 \%$ CI $0.77-1.08$ ) in the fully adjusted model. We found a strong positive, statistically significant association between night shift work (HR 12.0, 95\% CI 3.17-45.2) and diabetes mortality and weaker associations with evening (HR 2.94, 95\% CI 0.63-13.7) and rotating (HR 1.57, $95 \%$ CI $0.34-7.21)$ shifts. We found strong positive, statistically significant associations between mortality from Alzheimer's or dementia among nurses working evening (HR 4.28, 95\% CI 1.62-11.3) and rotating (HR 5.39, 95\% CI 2.35-12.3) shifts in the fully adjusted model. There was no evidence of association between working night shifts and Alzheimer's or dementia (HR 0.70, 95\% CI 0.09-5.72), but this analysis was based only on a single case. Finally, we found no evidence of an increased risk in mortality from psychiatric diseases.

We found no significant associations between shift work and mortality due to any of the cancer subtypes 
Table 2. Characteristics of 18015 nurses by working shift type at baseline (1993 and 1999).

\begin{tabular}{|c|c|c|c|c|c|c|c|c|c|}
\hline & \multicolumn{2}{|c|}{ Day } & \multicolumn{2}{|c|}{ Evening } & \multicolumn{2}{|c|}{ Night } & \multicolumn{2}{|c|}{ Rotating } & \multirow[t]{2}{*}{ P-value } \\
\hline & $\mathrm{N}$ & $\%$ & $\mathrm{~N}$ & $\%$ & $\mathrm{~N}$ & $\%$ & $\mathrm{~N}$ & $\%$ & \\
\hline Total & 11272 & 62.6 & 1805 & 10.0 & 980 & 5.4 & 3958 & 22.0 & \\
\hline Number of deaths ${ }^{b}$ & 934 & 57.8 & 236 & 14.6 & 151 & 9.3 & 295 & 18.3 & \\
\hline Person-years & 199327 & 62.9 & 32245 & 10.2 & 17662 & 5.6 & 67410 & 21.3 & \\
\hline \multicolumn{10}{|l|}{ Body mass index $\left(\mathrm{kg} / \mathrm{m}^{2}\right)$} \\
\hline$<18.5$ & 200 & 1.8 & 54 & 3.0 & 31 & 3.2 & 69 & 1.7 & $<0.001$ \\
\hline $18.5-24.9$ & 7945 & 70.5 & 1308 & 72.5 & 612 & 62.4 & 2823 & 71.3 & \\
\hline $25-29.9$ & 2494 & 22.1 & 342 & 18.9 & 257 & 26.2 & 871 & 22.0 & \\
\hline$\geq 30$ & 633 & 5.6 & 101 & 5.6 & 80 & 8.2 & 195 & 4.9 & \\
\hline \multicolumn{10}{|l|}{ Smoking status } \\
\hline Never & 4260 & 37.8 & 669 & 37.1 & 298 & 30.4 & 1498 & 37.8 & $<0.001$ \\
\hline Past & 3390 & 30.1 & 471 & 26.1 & 238 & 24.3 & 1145 & 28.9 & \\
\hline Current & 3622 & 32.1 & 665 & 36.8 & 444 & 45.3 & 1315 & 33.2 & \\
\hline \multicolumn{10}{|l|}{ Number of pack years ${ }^{c}$} \\
\hline$\leq 10$ & 3260 & 28.9 & 460 & 25.5 & 214 & 21.8 & 1162 & 29.4 & $<0.001$ \\
\hline $11-20$ & 1823 & 16.2 & 287 & 15.9 & 173 & 17.7 & 659 & 16.6 & \\
\hline$>20$ & 1929 & 17.1 & 389 & 21.6 & 295 & 30.1 & 639 & 16.1 & \\
\hline \multicolumn{10}{|l|}{ Alcohol consumption (drinks/week) } \\
\hline 0 & 1097 & 9.7 & 333 & 18.4 & 252 & 25.7 & 508 & 12.8 & $<0.001$ \\
\hline 1-14 (moderate) & 7312 & 64.9 & 1134 & 62.8 & 523 & 53.4 & 2553 & 64.5 & \\
\hline$>15$ (heavy) & 2863 & 25.4 & 338 & 18.7 & 205 & 20.9 & 897 & 22.7 & \\
\hline \multicolumn{10}{|l|}{ Physical activity } \\
\hline Low & 649 & 5.8 & 78 & 4.3 & 56 & 5.7 & 173 & 4.4 & $<0.001$ \\
\hline High & 3157 & 28.0 & 511 & 28.3 & 309 & 31.5 & 1176 & 29.7 & \\
\hline Medium & 7466 & 66.2 & 1216 & 67.4 & 615 & 62.8 & 2609 & 65.9 & \\
\hline \multicolumn{10}{|l|}{ Diet } \\
\hline Consume vegetables on daily basis & 11141 & 98.8 & 1773 & 98.2 & 962 & 98.2 & 3911 & 98.8 & 0.059 \\
\hline Consume fruit on daily basis & 10887 & 96.6 & 1711 & 94.8 & 939 & 95.8 & 3850 & 97.3 & $<0.001$ \\
\hline Avoids fatty meat & 10363 & 91.9 & 1619 & 89.7 & 862 & 88.0 & 3626 & 91.6 & $<0.001$ \\
\hline Self-reported pre-existing diseases & & & & & & & & & \\
\hline Hypertension & 1194 & 10.6 & 198 & 11.0 & 123 & 12.6 & 375 & 9.5 & 0.025 \\
\hline Diabetes & 91 & 0.8 & 23 & 1.3 & 17 & 1.7 & 27 & 0.7 & 0.003 \\
\hline Myocardial infarction & 26 & 0.2 & 9 & 0.5 & 5 & 0.5 & 7 & 0.2 & 0.052 \\
\hline Self-reported health & & & & & & & & & \\
\hline Very good & 5218 & 46.3 & 618 & 34.2 & 338 & 34.5 & 1820 & 46.0 & $<0.001$ \\
\hline Good & 4868 & 43.2 & 885 & 49.0 & 466 & 47.6 & 1721 & 43.5 & \\
\hline Moderate & 1075 & 9.5 & 271 & 15.0 & 169 & 17.2 & 379 & 9.6 & \\
\hline Bad & 100 & 0.9 & 28 & 1.6 & 7 & 0.7 & 31 & 0.8 & \\
\hline Very bad & 11 & 0.1 & 3 & 0.2 & 0 & 0.0 & 7 & 0.2 & \\
\hline Working status & & & & & & & & & \\
\hline Working & 11220 & 99.5 & 1797 & 99.6 & 979 & 99.9 & 3928 & 99.2 & 0.34 \\
\hline Homeworker & 0 & 0.0 & 0 & 0.0 & 0 & 0.0 & 1 & $<0.1$ & \\
\hline Retired & 6 & 0.1 & 1 & 0.1 & 0 & 0.0 & 2 & 0.1 & \\
\hline Unemployed/rehabilitation & 2 & $<0.1$ & 1 & 0.1 & 0 & 0.0 & 1 & $<0.1$ & \\
\hline Other & $4 \overline{4}$ & 0.4 & 6 & 0.3 & 1 & 0.1 & 26 & 0.7 & \\
\hline Stressful work environment & & & & & & & & & \\
\hline Never & 141 & 1.3 & 29 & 1.6 & 41 & 4.2 & 40 & 1.0 & $<0.001$ \\
\hline Rarely & 1530 & 13.6 & 362 & 20.1 & 383 & 39.1 & 572 & 14.5 & \\
\hline Occasionally & 4643 & 41.2 & 904 & 50.1 & 433 & 44.2 & 1808 & 45.7 & \\
\hline Often & 3868 & 34.3 & 445 & 24.7 & 108 & 11.0 & 1292 & 32.6 & \\
\hline Almost always & 1090 & 9.7 & 65 & 3.6 & 15 & 1.5 & 246 & 6.2 & \\
\hline Marital status & & & & & & & & & \\
\hline Married & 8602 & 76.3 & 1345 & 74.5 & 665 & 67.9 & 2864 & 72.4 & $<0.001$ \\
\hline Separated & 185 & 1.6 & 32 & 1.8 & 18 & 1.8 & 93 & 2.3 & \\
\hline Divorced & 1223 & 10.8 & 214 & 11.9 & 151 & 15.4 & 506 & 12.8 & \\
\hline Single & 832 & 7.4 & 126 & 7.0 & 80 & 8.2 & 367 & 9.3 & \\
\hline Widow & 430 & 3.8 & 88 & 4.9 & 66 & 6.7 & 128 & 3.2 & \\
\hline Use of hormone therapy & & & & & & & & & \\
\hline Never-users & 8574 & 76.1 & 1293 & 71.6 & 672 & 68.6 & 3125 & 79.0 & $<0.001$ \\
\hline Ever-users & 2698 & 23.9 & 512 & 28.4 & 308 & 31.4 & 833 & 21.0 & \\
\hline Use of oral contraceptives & & & & & & & & & \\
\hline Never-users & 3580 & 31.8 & 723 & 40.1 & 412 & 42.0 & 1073 & 27.1 & $<0.001$ \\
\hline Ever-users & 7692 & 68.2 & 1082 & 59.9 & 568 & 58.0 & 2885 & 72.9 & \\
\hline Births & & & & & & & & & \\
\hline 0 & 1213 & 10.8 & 191 & 10.6 & 117 & 11.9 & 457 & 11.5 & 0.38 \\
\hline$\geq 1$ & 10059 & 89.2 & 1614 & 89.4 & 863 & 88.1 & 3501 & 88.5 & \\
\hline
\end{tabular}

a Categorical groups compared using Pearson's Chi-squared.

${ }^{\mathrm{b}}$ All-cause mortality.

c One pack year was defined as 20 cigarettes/day/year in ever-smokers. 
Table 5. Association between shift work and cause-specific mortality. [HR=hazard ratio; $95 \% \mathrm{Cl}=95 \%$ confidence intervals; ref=reference]

\begin{tabular}{|c|c|c|c|c|c|c|}
\hline \multirow[t]{2}{*}{ Mortality } & \multicolumn{2}{|c|}{ Cases } & \multicolumn{2}{|c|}{ Crude a } & \multicolumn{2}{|c|}{ Adjusted ${ }^{b}$} \\
\hline & $\mathrm{N}$ & $\%$ & $\mathrm{HR}$ & $95 \% \mathrm{Cl}$ & $\mathrm{HR}$ & $95 \% \mathrm{Cl}$ \\
\hline \multicolumn{7}{|c|}{ Ischemic heart disease } \\
\hline Day shifts (ref) & 29 & 46.0 & 1.00 & & 1.00 & \\
\hline Evening shifts & 11 & 17.5 & 2.30 & $1.15-4.60$ & 1.71 & $0.84-3$. \\
\hline Night shifts & 11 & 17.5 & 4.10 & $2.05-8.22$ & 2.30 & $1.07-4.9$ \\
\hline Rotating shifts & 12 & 19.1 & 1.28 & $0.65-2.51$ & 1.22 & $0.61-2$ \\
\hline \multicolumn{7}{|l|}{ Hypertension } \\
\hline Day shifts (ref) & 19 & 47.5 & 1.00 & & 1.00 & \\
\hline Evening shifts & 5 & 12.5 & 1.57 & $0.59-4.22$ & 1.60 & $0.57-4$. \\
\hline Night shifts & 6 & 15.0 & 3.30 & $1.32-8.28$ & 2.35 & $0.86-6.3$ \\
\hline Rotating shifts & 10 & 25.0 & 1.68 & $0.78-3.62$ & 2.04 & $0.92-4$. \\
\hline \multicolumn{7}{|l|}{ Stroke } \\
\hline Day shifts (ref) & 42 & 56.8 & 1.00 & & 1.00 & \\
\hline Evening shifts & 7 & 9.5 & 1.00 & $0.45-2.23$ & 0.91 & $0.40-2.0$ \\
\hline Night shifts & 9 & 12.2 & 2.27 & $1.10-4.67$ & 1.98 & $0.92-4.2$ \\
\hline Rotating shifts & 16 & 2.6 & 1.20 & $0.68-2.14$ & 1.24 & $0.70-2.2$ \\
\hline \multicolumn{7}{|c|}{ Other cardiovascular } \\
\hline Day shifts (ref) & 33 & 53.2 & 1.00 & & 1.00 & \\
\hline Evening shifts & 14 & 22.6 & 2.55 & $1.37-4.77$ & 2.25 & $1.18-4.3$ \\
\hline Night shifts & 4 & 6.5 & 1.29 & $0.46-3.64$ & 0.95 & $0.32-2.80$ \\
\hline Rotating shifts & 11 & 17.7 & 1.05 & $0.53-2.08$ & 1.19 & $0.59-2.3$ \\
\hline
\end{tabular}

a Model adjusted for age.

${ }^{\mathrm{b}}$ Model adjusted for age, smoking, pack-years, physical activity, body mass index $\left(\mathrm{kg} / \mathrm{m}^{2}\right)$, alcohol consumption, diet (vegetables, fruit and fatty meat consumption), pre-existing diseases (hypertension, diabetes and myocardial infarction), self-reported health, stressful work environment, marital status, female reproductive factors (birth, use of hormone therapy, and oral contraceptives).

cause mortality among male night shift workers, which may suggest different susceptibility by gender. Nätti et al's observed gender differences were consistent with Åkerstedt et al's results, where a significant association with mortality was limited to female white-collar night shift workers, whereas none was found among male night workers or female blue-collar workers (10). Gender variation in susceptibility to night work may also explain why our results conflict with studies based on male participants $(3,4,7,8,12)$.

Work schedule is dependent on age, and it has previously been documented that nurses in younger age groups are more likely to work in rotating shifts, whereas nurses $>40$ years more often work in day or evening shifts (30). However, $90 \%$ of nurses working in Denmark have worked night shifts at some point, typically early in their career after completing their training. We found a similar increase in all-cause mortality for night (26\%) and evening (29\%) shift workers. This might be explained by the fact that a large number of nurses who worked evening shifts at the time of cohort recruitment have worked night shifts earlier in their career. Another possible explanation for the increased mortality among evening workers might be that this work schedule is associated with an increase in social stress and more work-family related conflicts. Furthermore, other unmeasured potentially confounding variables such as weekly working hours could explain these findings.

The lack of association between rotating shift work and all-cause mortality is possibly explained by the fact that rotating shift work involves day, evening and night shifts or only day and evening shifts and few night shifts in a sequence, probably resulting in minor circadian disturbance. A Danish report on occupational health among nurses from the Danish Nurses Organization, reveals that $43 \%$ of nurses aged $20-29$ years work rotating 3 -shifts, while this attenuates to $20 \%$ among nurses aged 40-49 years and $12 \%$ among those aged $50-59$ years, indicating that 3 -shift rotating work is more prevalent early in the career, and thus making it less prevalent in our cohort (30). Alternatively, the lack of association between rotating shifts and all-cause mortality might be explained if a high proportion of previous rotating shift workers changed to day shifts positions. This could result in increased mortality rate among day workers and an underestimation of the impact of shift work (30).

The IARC has classified shift work that involves circadian disruption as "probably carcinogenic to humans" (group 2A) due to increased risk of breast cancer incidence among women (31). We found no association between shift work and all-cancer mortality, in agreement with $\mathrm{Gu}$ et al (6), and in contrast to Nätti et al (5). Furthermore, no evidence of an increase in mortality related to shift work was found for any specific cancers including cancer of the breast, which is in agreement with some of the previous studies $(6,7,17)$. However our findings are in conflict with increased mortality due to ovarian cancer reported by Carter et al (19) and increased mortality due to lung cancer ( $\geq 15$ years of rotating night shift work) and breast cancer reported by $\mathrm{Gu}$ et al (6). Gu et al. found an association with breast cancer mortality only among those working night shifts $\geq 30$ years (HR $1.47,95 \% \mathrm{CI}$ 0.94-2.32), and none with more recent exposures, suggesting the relevance of early exposure for the development for breast cancer (6). This in line with Menegaux et al (32) who found the strongest risk of developing breast cancer among women who worked night shifts for $>4$ years before their first full-term pregnancy, a period where mammary glands are not completely differentiated and possibly more susceptible to circadian disruption effects. Thus lack of effects of shift work on breast cancer mortality in our study may be due, at least in part, to lack of information on shift work duration and shift work schedules, before the first childbirth.

We found a $71 \%$ increase in CVD mortality among nurses working night shifts, which was considerably higher than the $19 \%$ and $23 \%$ detected in American nurses with $\geq 5$ and $\geq 15$ years of night shift work, respectively (6). We furthermore found the highest increased rate $(130 \%)$ related to night shift work for IHD mortality, which is consistent with some studies $(6,33,34)$, 
however is inconsistent with others $(8,35,36)$. Inconsistencies are likely explained by differences in study populations in terms of the gender and age of included subjects. Knutsson et al's case-control study of MI incidence detected associations with both male and female shift workers, but found substantially higher risk [odds ratio (OR) 3.0, 95\% CI 1.4-6.5] among females aged $45-55$ years than males of the same age (OR 1.6, 95\% C $1.1-2.4)$ (37). Since the majority ( $80 \%)$ of participants included were $44-55$ years of age at the time of recruitment, a large proportion of the shift-working nurses in our study might, based on the results of Knutsson et al, be at particularly high risk of developing MI due to shift work, possibly explaining strong effects observed in our study and the lack of association between shift work and IHD mortality in most studies based on male participants $(8,35,36)$. We also found statistically nonsignificant associations between night shift work and risk of dying from hypertension and stroke and increased risk of dying from other CVD among evening shift workers. These findings agree with Vyas et al's recent meta-analysis that has linked shift work to increases in the incidence of various vascular events including, MI $(23 \%)$, ischemic stroke (5\%) and coronary events $(24 \%)$ (20). Disruption of circadian rhythms is thought to be the plausible mechanisms linking shift work with CVD through multifactorial pathways $(38,39)$, involving weight gain, physical inactivity, development of type II diabetes, and other pathways (38).

The highest rate ratio associated with night shift work in this study was observed for diabetes mortality, however, the results are based on a limited number of diabetes deaths $(\mathrm{N}=8)$ and therefore somewhat unstable. Our results are considerably stronger than the effect in the Karlsson et al (12) study of Swedish male workers from the pulp and paper industry (HR $1.24,95 \%$ CI $0.91-1.70$ ). Karlsson et al included males enrolled at age 10 in 1952 and used shift work as a dichotomized exposure (all shift versus day worker), but, on the other hand, included data on duration of shift work and found strong indication of a positive dose-response relationship with diabetes mortality. We have recently reported a strong significant association between night shift work and diabetes incidence (HR $1.58,95 \%$ CI $1.25-1.99$ ) in the DNC (12). Results based on the Nurses' Health Study I and II (40) and the Black Women's Health Study (41) also detected an association between night shift work and diabetes incidence.

We provided novel results of the strong increase in mortality from Alzheimer's and dementia among nurses working evening and rotating shifts. To our knowledge this is the first study to report such an association. However, our results are based on a limited number of cases ( 33 deaths in total), and further studies of this issue are clearly warranted. Studies have put forth plausible biological mechanisms suggesting that sleep disturbance results in substantial detrimental cognitive effects (26). Unlike for all-cause mortality where no effect of rotating shifts was observed, we found the strongest associations for Alzheimer's and dementia among rotating shifts workers. This result may suggest relevance of other mechanisms, possibly stress-related for Alzheimer's and dementia than for all-cause mortality where circadian disruption may be more relevant. Furthermore, recent studies have shown an association between short sleep duration with greater $\beta$-amyloid $(\mathrm{A} \beta)$ burden, a biomarker of Alzheimer's progression (25), and that better sleep consolidation substantially attenuated the negative effects of the apolipoprotein E $\varepsilon 4$ allele, a common genetic risk factor for Alzheimer's (24).

\section{Strengths and limitations}

A major strength of this study is the utilization of a nationwide prospective cohort of 18015 female nurses, with detailed information on work schedules (night, evening, rotating, day), lifestyle, BMI, history of diseases, and reproductive factors at the time of cohort enrolment, and long follow-up for overall and cause-specific mortality in a national registry. In contrast to the commonly used shift work definition as a dichotomous exposure, typically comparing day to shift workers and combining evening, night and rotating shifts, in this study we were able to separate effect of night, evening and rotating shift in comparison to day shift work for the first time in a study of mortality. We excluded $10716(\sim 37 \%)$ of the participants due to missing values. A majority of the participants (6721) were excluded because the question about work schedule was aimed at nurses working $(74 \%)$ at the time of recruitment only. We found that the age-adjusted mortality was significantly higher among excluded nurses (HR 4.07, 95\% CI 3.84-4.31) compared to included nurses, as expected since excluded nurses were on average 10 years older (mean age 60.0 years versus 50.2 years) at the time of recruitment. This is explained by the fact that a majority of excluded nurses were retired or on sick leave or disability retirement at the time of recruitment, and were therefore not included in the assessment of shift work. We found no major differences in lifestyle, BMI, or other characteristics between included and excluded nurses (supplemental material tables A and B, http://www.sjweh.fi/index.php?page=data-repository).

A major limitation of this study is the lack of data on intensity and duration of shift work in terms of the number of shifts per week or month, number of working hours per shift, and number of years working in shift work. The exposure was only assessed at baseline with no follow-up on changes in work schedule, potentially introducing exposure misclassification. However the nurses were followed from age 44 years when in general it is less likely for nurses to change work schedule than 
earlier in their careers. We also lacked information on duration of recovery periods and work schedules early in their career (from completion of professional training to cohort recruitment) and information on different types of rotating shift schedule, particularly whether or not rotating shifts involved night shifts. Another limitation is the small number of deaths for some of the examined outcomes (eg, diabetes and Alzheimer's and dementia), resulting in wide confidence intervals, and our findings need to be replicated in larger studies. Furthermore, the registration of underlying and contributory causes of death relies entirely on the physician responsible for completing the death certificate, without central validation, which together with increasing diagnostic facilities influence the accuracy and correctness of the register and changes in mortality rates over the years (29).

Healthcare professionals are generally considered to be healthier than the general population, and a "healthy worker effect" might have biased our results. Nurses participating in this cohort have been found to be healthier than Danish women in general, as they smoked less and had higher levels of physical activity, but on average they consumed more alcohol $(27,42)$. However, there were no major health differences between nurses and the rest of Danish female population in use of healthcare and disease occurrence $(27,42)$.

In conclusion, we found evidence of an increased all-cause mortality risk among female nurses working in night or evening shifts, compared to those working in day shifts. We further found evidence of increased mortality due to CVD and diabetes, and Alzheimer's and dementia, while there was no evidence of an increased risk in mortality from psychiatric diseases and overall or cause specific cancer. Additional studies of mortality among shift workers are warranted.

\section{Acknowledgements}

The authors declare no conflicts of interest.

\section{Ethical approval}

Relevant Danish ethical committees and Danish Data Protection Agency approved this study (j.nr. 201541-4307), and participants provided written informed consent at recruitment.

\section{References}

1. Hughes V. Health Risks Associated with Nurse Night Shift Work: A Systematic Review. GSTF J Nurs Heal Care.
2015;2:39-44. https://doi.org/10.5176/2010-4804_2.2.74.

2. Wang XS, Armstrong MEG, Cairns BJ, Key TJ, Travis RC. Shift work and chronic disease: The epidemiological evidence. Occup Med (Chic Ill). 2011;61:78-89. https://doi. org/10.1093/occmed/kqr001.

3. Taylor PJ, Pocock SJ. Mortality of shift and day workers 195668. Br J Ind Med. 1972;29:201-7. https://doi.org/10.1136/ oem.29.2.201.

4. Knutsson A, Hammar N, Karlsson B. Shift workers' mortality scrutinized. Chronobiol Int. 2004;21:1049-53. https://doi. org/10.1081/CBI-200035942.

5. Natti J, Anttila T, Oinas T, Mustosmaki A. Night work and mortality: prospective study among Finnish employees over the time span 1984 to 2008. Chronobiol Int. 2012;29:601-9. https://doi.org/10.3109/07420528.2012.675262.

6. Gu F, Han J, Laden F, Pan A, Caporaso NE, Stampfer MJ, et al. Total and cause-specific mortality of U.S. nurses working rotating night shifts. Am J Prev Med. Elsevier; 2015;48:241-52.

7. Yong M, Nasterlack M, Messerer P, Oberlinner C, Lang S. A retrospective cohort study of shift work and risk of cancerspecific mortality in German male chemical workers. Int Arch Occup Environ Health. 2014;87:175-83. https://doi. org/10.1007/s00420-013-0843-3.

8. Bøggild H, Suadicani P, Hein HO, Gyntelberg F. Shift work, social class, and ischaemic heart disease in middle aged and elderly men; a 22 year follow up in the Copenhagen Male Study. Occup Environ Med. 1999;56:640-5. https://doi. org/10.1136/oem.56.9.640.

9. Shives B, Riley B. Towards evidence based emergency medicine: best BETs from the Manchester Royal Infirmary. BET 2: Is shift work bad for you? Emerg Med J. 2013;30:859.

10. Akerstedt T, Kecklund G, Johansson S-E. Shift work and mortality. Chronobiol Int. 2004;21:1055-61. https://doi. org/10.1081/CBI-200038520.

11. Lin X, Chen W, Wei F, Ying M, Wei W, Xie X. Night-shift work increases morbidity of breast cancer and all-cause mortality: A meta-analysis of 16 prospective cohort studies. Sleep Med [Internet]. 2015;16:1381-7. https://doi.org/10.1016/j. sleep.2015.02.543.

12. Karlsson B, Alfredsson L, Knutsson A, Andersson E, Torén K. Total mortality and cause-specific mortality of Swedish shiftand dayworkers in the pulp and paper industry in 1952-2001. Scand J Work Environ Health. 2005;31:30-5. https://doi. org/10.5271/sjweh.845.

13. Lin X, Chen W, Wei F, Ying M, Wei W, Xie X. Night-shift work increases morbidity of breast cancer and all-cause mortality: A meta-analysis of 16 prospective cohort studies. Sleep Med. 2015;16:1381-7. https://doi.org/10.1016/j. sleep.2015.02.543.

14. Bøggild H, Suadicani P, Hein HO, Gyntelberg F. Shift work, social class and ischemic heart disease in middle-aged and elderly men. A 22-year follow-up in the "Copenhagen Male Study." Ugeskr Laeger. 2000;162:1882-6.

15. International Agency for Research on Cancer (IARC). IARC Working Group on the Evaluation of Carcinogenic Risks to 
Humans. Tobacco smoke and involuntary smoking. IARC Work Gr Eval Carcinog Risks to Humans. 2007;98.

16. Stevens RG, Brainard GC, Blask DE, Lockley SW, Motta ME. Breast Cancer and Circadian Disruption From Electric Lighting in the Modern World. CA Cancer J Clin. 2014;64:207-18. https://doi.org/10.3322/caac.21218.

17. Lin Y, Ueda J, Yagyu K, Kurosawa M, Tamakoshi A, Kikuchi S. A prospective cohort study of shift work and the risk of death from pancreatic cancer in Japanese men. Cancer causes Control. 2013;24:1357-61. https://doi.org/10.1007/s10552013-0214-0.

18. Lin Y, Nishiyama T, Kurosawa M, Tamakoshi A, Kubo T, Fujino Y, et al. Association between shift work and the risk of death from biliary tract cancer in Japanese men. BMC Cancer. BMC Cancer; 2015;15:757. https://doi.org/10.1186/s12885015-1722-y.

19. Carter BD, Ryan Diver W, Hildebrand JS, Patel A V., Gapstur SM. Circadian disruption and fatal ovarian cancer. Am J Prev Med. Elsevier; 2014;46:S34-41.

20. Vyas M V., Garg a. X, Iansavichus a. V., Costella J, Donner A, Laugsand LE, et al. Shift work and vascular events: systematic review and meta-analysis. Bmj. 2012;345:e4800-e4800. https://doi.org/10.1136/bmj.e4800.

21. Vetter C, Devore EE, Wegrzyn LR, Massa J, Speizer FE, Kawachi I, et al. Association Between Rotating Night Shift Work and Risk of Coronary Heart Disease Among Women. Jama. 2016;315:1726-34. https://doi.org/10.1001/ jama.2016.4454.

22. Hansen AB, Stayner L, Hansen J, Andersen ZJ. Night shift work and incidence of diabetes in the Danish Nurse Cohort. Occup Environ Med. 2016 Apr;73:262-8. https://doi. org/10.1136/oemed-2015-103342.

23. Frost P, Kolstad HA, Bonde JP. Shift work and the risk of ischemic heart disease - a systematic review of the epidemiologic evidence. Scand J Work Environ Health. 2009;35:163-79. https://doi.org/10.5271/sjweh.1319.

24. Lim ASP, Yu L, Kowgier M, Schneider JA, Buchman AS, Bennett DA, et al. Modification of the Relationship of the Apolipoprotein E $\varepsilon 4$ Allele to the Risk of Alzheimer Disease and Neurofibrillary Tangle Density by Sleep. JAMA Neurol. American Medical Association; 2013;70:1977-81. https://doi. org/10.1001/jamaneurol.2013.4215.

25. Spira AP, Gamaldo AA, An Y, Wu MN, Simonsick EM, Bilgel $M$, et al. Self-reported Sleep and $\beta$-Amyloid Deposition in Community-Dwelling Older Adults. JAMA Neurol. American Medical Association; 2013;70:86-93. https://doi.org/10.1001/ jamaneurol.2013.4258.

26. Walker MP. Cognitive consequences of sleep and sleep loss. Sleep Med. 2008;9 Suppl 1:S29-34. https://doi.org/10.1016/ S1389-9457(08)70014-5.

27. Hundrup YA, Simonsen MK, Jørgensen T, Obel EB. Cohort profile: The danish nurse cohort. Int $\mathrm{J}$ Epidemiol. 2012;41:1241-7. https://doi.org/10.1093/ije/dyr042.

28. Pedersen CB. The Danish Civil Registration System. Scand J Public Health. 2011;39:22-5. https://doi. org/10.1177/1403494810387965.

29. Helweg-Larsen K. The Danish Register of Causes of Death. Scand J Public Health. 2011;39:26-9. https://doi. org/10.1177/1403494811399958

30. Wethje A, Borg V. Sygeplejerskers arbejdsmiljø, trivsel og helbred [Internet]. 2005 [cited 2016 Jul 5]. p. 1-31. Available from: www.dsr.dk.

31. IARC Working Group on the Evaluation of Carcinogenic Risks to Humans. Painting, firefighting, and shiftwork. IARC Monogr Eval Carcinog Risks Hum. 2010;98:9-764.

32. Menegaux F, Truong T, Anger A, Cordina-Duverger E, Lamkarkach F, Arveux P, et al. Night work and breast cancer: A population-based case-control study in France (the CECILE study). Int J Cancer. 2012;132:924-31. https://doi. org/10.1002/ijc.27669.

33. Fujino Y, Iso H, Tamakoshi A, Inaba Y, Koizumi A, Kubo $\mathrm{T}$, et al. A prospective cohort study of shift work and risk of ischemic heart disease in Japanese male workers. Am J Epidemiol. United States; 2006 Jul;164:128-35.

34. Kawachi I, Colditz GA, Stampfer MJ, Willett WC, Manson JE, Speizer FE, et al. Prospective study of shift work and risk of coronary heart disease in women. Circulation. UNITED STATES; 1995 Dec;92:3178-82.

35. McNamee R, Binks K, Jones S, Faulkner D, Slovak A, Cherry NM. Shiftwork and mortality from ischaemic heart disease. Occup Env Med. 1996;53:367-73. https://doi.org/10.1136/ oem.53.6.367.

36. Yadegarfar G, McNamee R. Shift work, confounding and death from ischaemic heart disease. Occup Environ Med. 2008;65:158-63. https://doi.org/10.1136/oem.2006.030627.

37. Knutsson A, Hallquist J, Reuterwall C, Theorell T, Akerstedt T. Shiftwork and myocardial infarction: a case-control study. Occup Environ Med. ENGLAND; 1999 Jan;56:46-50.

38. Puttonen S, Härmä M, Hublin C. Shift work and cardiovascular disease - Pathways from circadian stress to morbidity. Scand J Work Environ Heal. 2010;36:96-108. https://doi.org/10.5271/ sjweh.2894.

39. Bøggild H, Knutsson A. Shift work, risk factors and cardiovascular disease. Scand J Work Environ Health. 1999 Apr;25:85-99. https://doi.org/10.5271/sjweh.410.

40. Pan A, Schernhammer ES, Sun Q, Hu FB. Rotating night shift work and risk of type 2 diabetes: two prospective cohort studies in women. PLoS Med. 2011;8:e1001141. https://doi. org/10.1371/journal.pmed.1001141.

41. Vimalananda VG, Palmer JR, Gerlovin H, Wise LA, Rosenzweig JL, Rosenberg L, et al. Night-shift work and incident diabetes among African-American women. Diabetologia. Springer Berlin Heidelberg; 2015 Apr 14;58:699-706.

42. Friis K, Ekholm O, Hundrup YA. Comparison of lifestyle and health among Danish nurses and the Danish female population: Is it possible to generalize findings from nurses to the general female population? Scand J Caring Sci. 2005;19:361-7. https://doi.org/10.1111/j.1471-6712.2005.00366.x.

Received for publication: 18 August 2016 